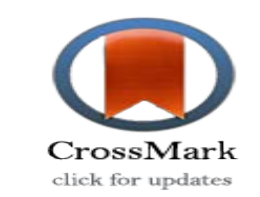

\title{
Comparison of coping strategies and perception of aging in successful and unsuccessful elderly people of ardabil in 2019
}

\author{
* Fereshteh Pourmohseni Koluri ${ }^{1}$, Maryam Seyed Kargar ${ }^{2}$, Eisa Jafari ${ }^{3}$, Sanaz \\ Eyni $^{4}$
}

1- Associate Professor, Department of Psychology, Payame Noor University, Tehran, Iran (Corresponding Author)

Email: fpmohseni@yahoo.com)

2- MA of Psychology, Payame Noor University, Tehran, Iran.

3- Associate Professor, Department of Psychology, Payame Noor University, Tehran, Iran.

4- PhD of psychology., Department of Psychology, Faculty of Educational Sciences and Psychology, University of Mohaghegh Ardabili, Ardebil, Iran.

\section{Abstract}

Introduction: Achieving the goals of successful ageing is considered to be an effective strategy for the promotion of health in the elderly. The purpose of present study was to compare the coping strategies and perception of aging in successful and unsuccessful elderly people in Ardabil city.

Methods: This study is a causal-comparative study. The statistical population consisted of elderly people in Ardebil who were selected through convenience sampling from 60 elderly people (30 successful elders and 30 unsuccessful elders) and were assessed with Baltes questionnaire for coping strategies (selection, optimization and compensation), and Barkers age perception questionnaire. Data analysis was performed using multivariate analyses of variance and U Mann Whitney test and through SPSS v.21 statistical software.

Results: The results showed that the successful elderly, as compared to the unsuccessful elderly, reported more favorable copnig strategies $(\mathrm{U}=244, \mathrm{P}<0.002)$. The results also showed that successful seniors had more perception of aging than compared to unsuccessful elderly people $(\mathrm{F}=15.19, \mathrm{P}<0.01)$.

Conclusions: The results of this study showed that successful seniors, compared with unsuccessful elderly people, are more likely to have copnig strategies and perception of aging.
\end{abstract}

Keywords: Coping Strategied, Perception of Aging, Successful Aging. 


\section{مقايسه راهبردهاى مقابله اي و ادراك از ييرى در سالمندان موفق و ناموفق شهر اردبيل در سال irgr}

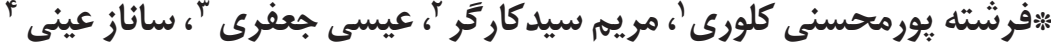

1- دانشيار كَروه روانشناسى، دانشكاه يِيام نور، تهران، ايران (نويسنده مسئول) يست الكترونيكى: yahoo.com r- كارشناس ارشد روانشناسى، دانشخاه يِيام نور، تهران، ايران.

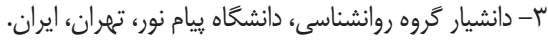

fا- دكتراى روانشناسى تخصصى، دانشكده علوم تربيتى و روانشناسى، دانشكاه محقق اردبيلى، اردبيل، ايران.

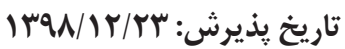

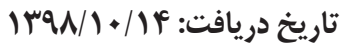

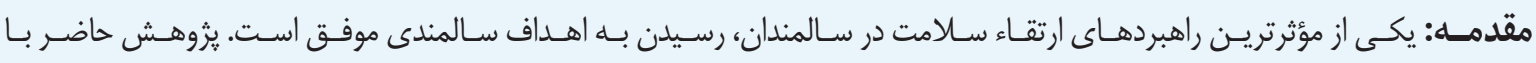

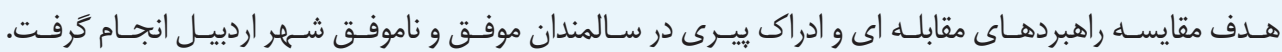

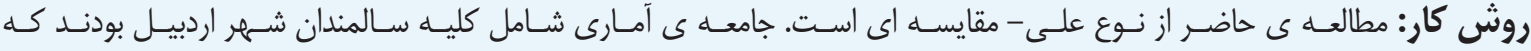

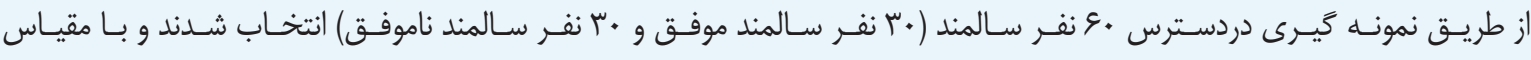

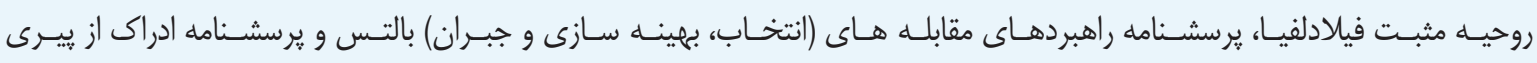

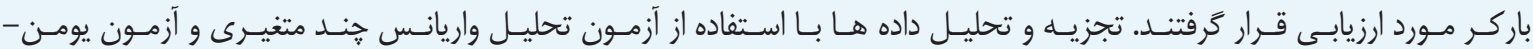

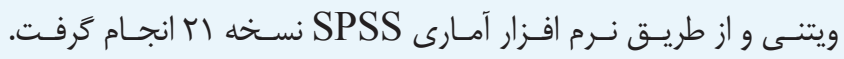

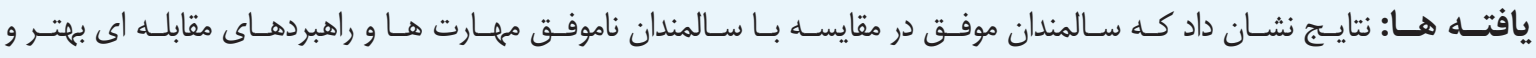

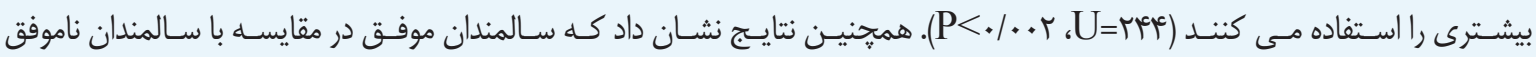

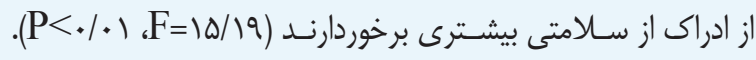

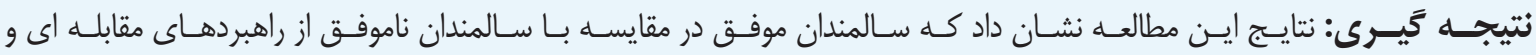

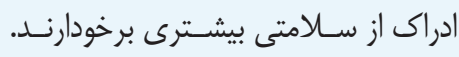
كليدوازه ها: راهبردهاى مقابله، ادراك از يبيرى، سالمندى موفق. برديس.

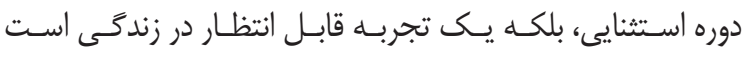

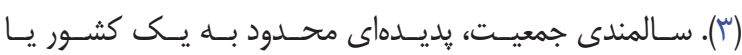

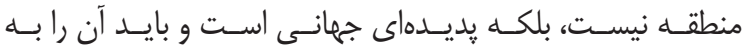

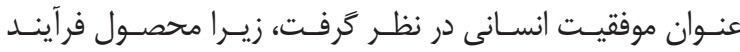

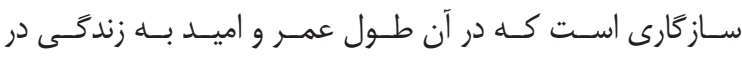

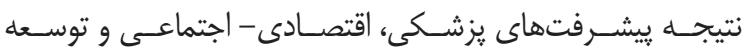

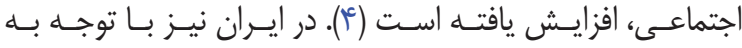

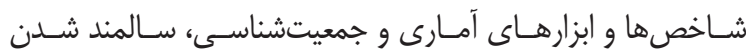

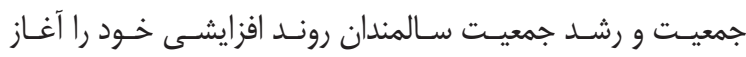

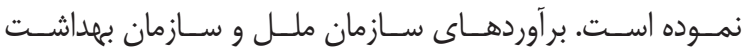

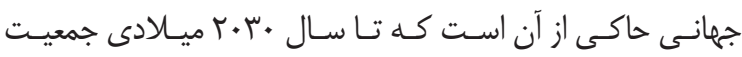

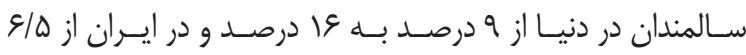

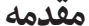

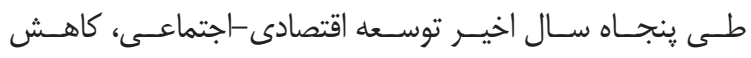

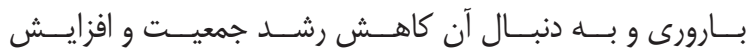

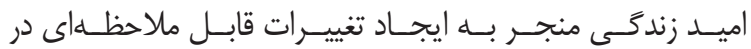

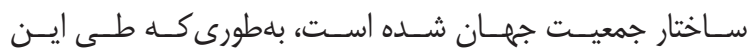

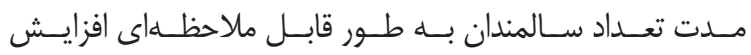
يافتــهـ اســت (1). در حــال حاضــر جمعيـت بــالاى •ع ســال

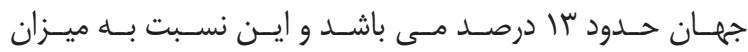

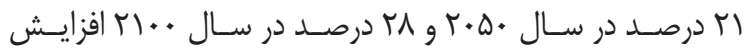
خواهـــ يافـت (r). جمعيتشناسـان حتـى افزايـش بيشـتر در طول

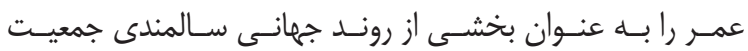

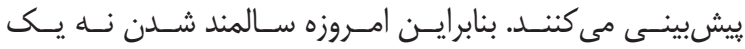


ايـن الخَـو، ســالمندى موفـق، فرآينـــى روانشــاختى اسـت كـهـ

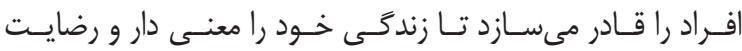

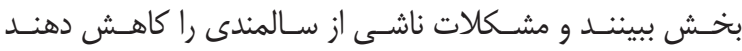

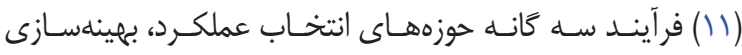

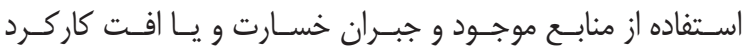

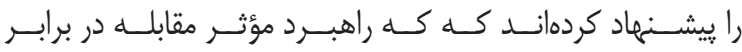

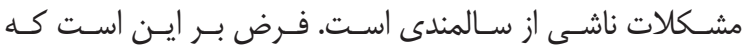

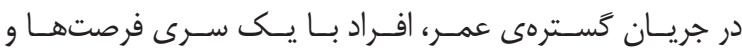

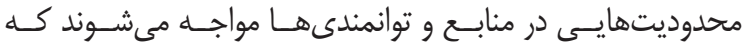

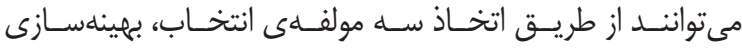

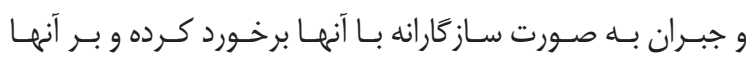

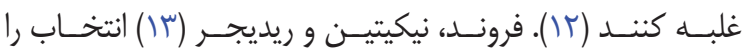

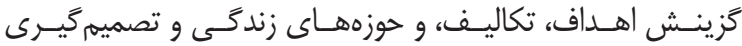

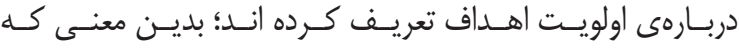

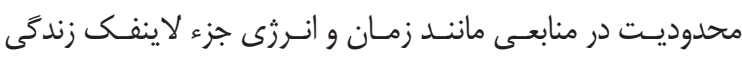

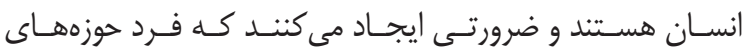

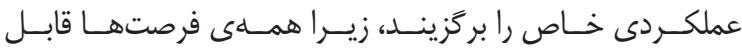

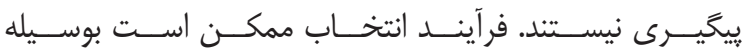

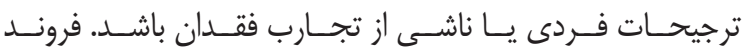

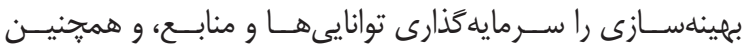

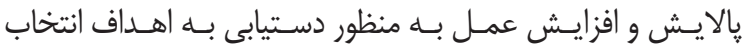

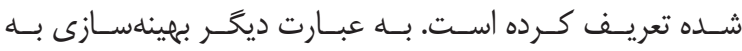

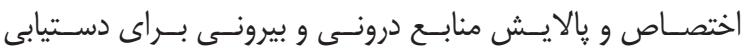

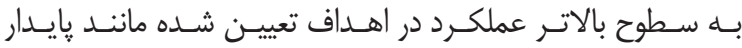

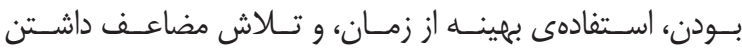

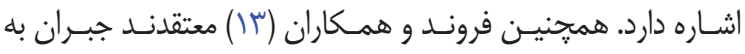

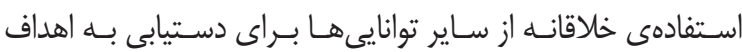

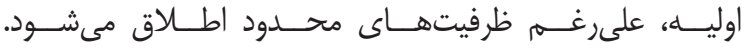

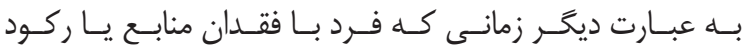

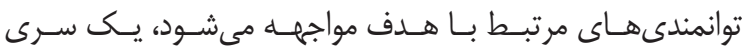

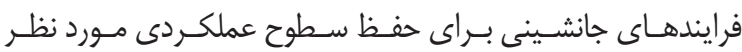

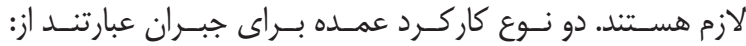

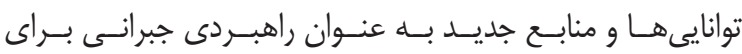

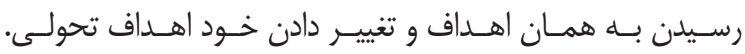

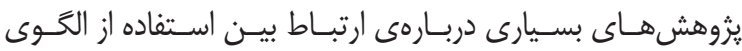

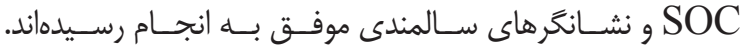

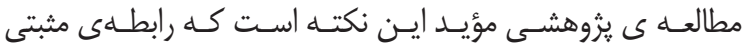

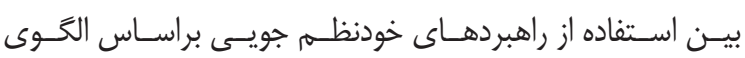

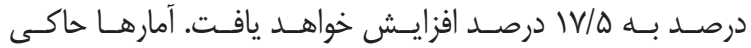

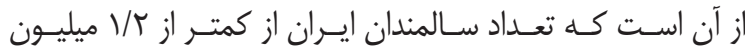

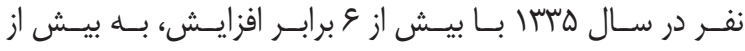

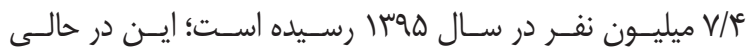
اسـت كـهـ جمعيـت كشـور در طـى ايـن مـــت كمـى بيشـتر از

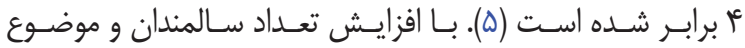

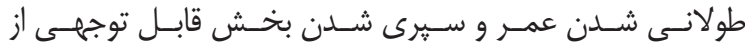

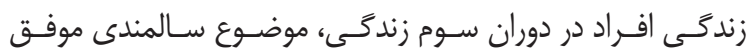

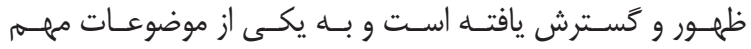

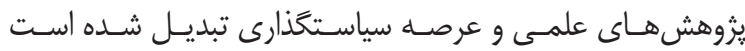

سـالمندى موفـق مفهومـى مهــمر اسـت كـه موضـوع يثوهشهاى زيـادى بـوده اسـت. تعـدادى از اصطلاحـات مشـابه، بــه صـورت

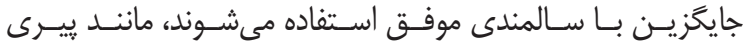

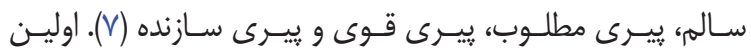

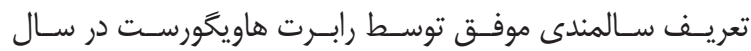

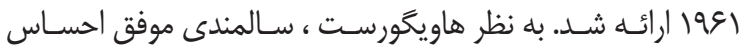

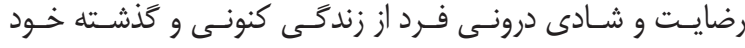

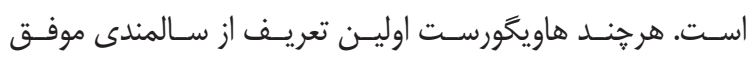

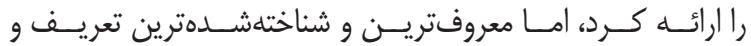
مــدل از ســالمندى موفـق را روو و كان در ســال I9AV ارائــهـ

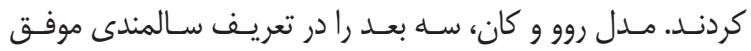

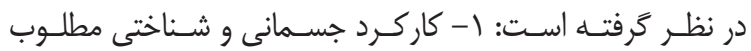

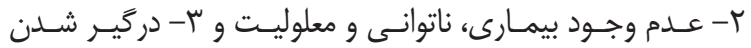

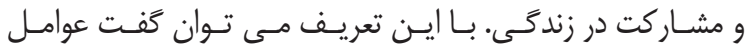

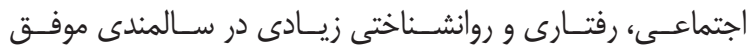

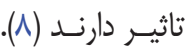

يكى از عواملى كـه در سـالمندى موفـق موثـر اسـت، راهبردهاى

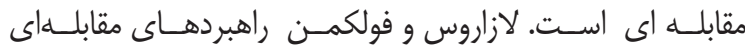

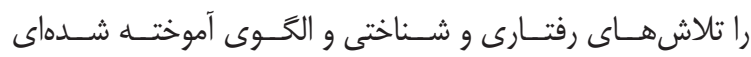

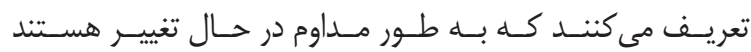

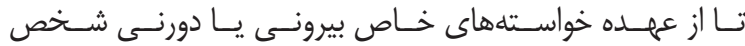

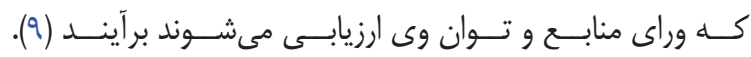

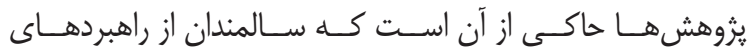

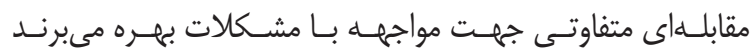
(•). در هميـن راسـتا، يزوهشهــاى سالمندشناسـى تعـدادى از مواز

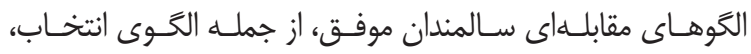

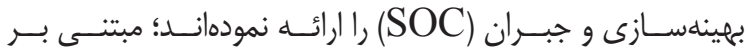




\section{فرشته يور محسنى كلورى هو هكاران}

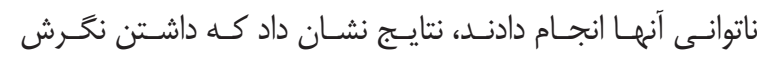

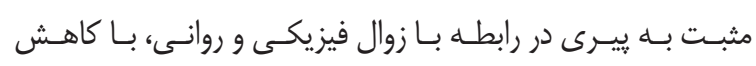

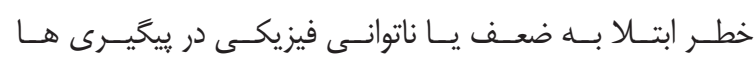
همـراه بـود.

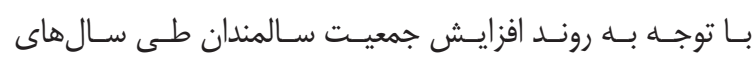

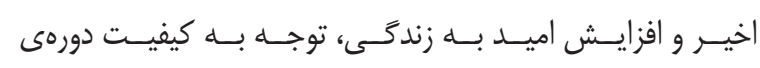

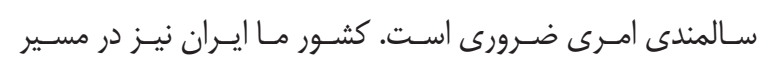

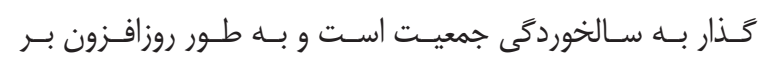

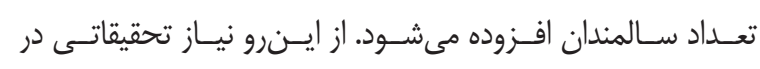

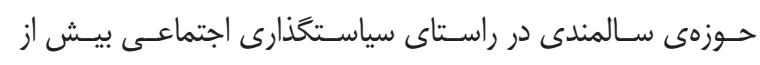

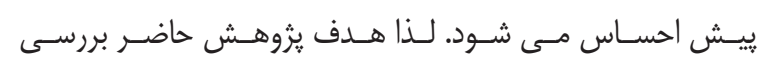

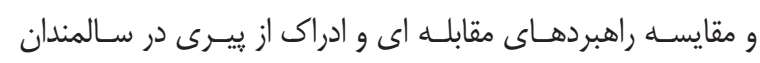

$$
\text { موفق و ناموفـق بـود. }
$$

\section{روش كار}

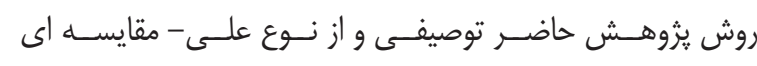

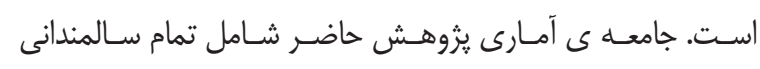

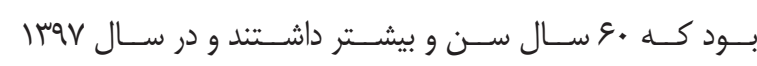

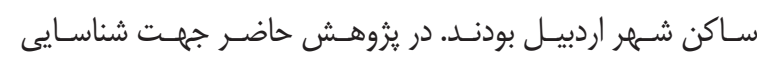

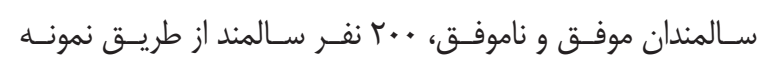

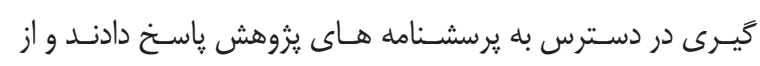

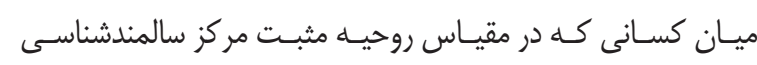

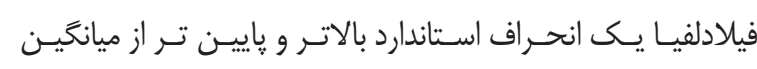

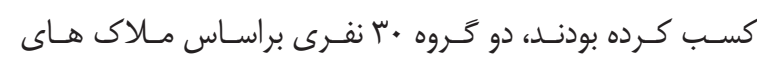

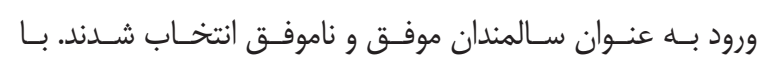

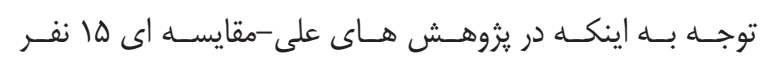

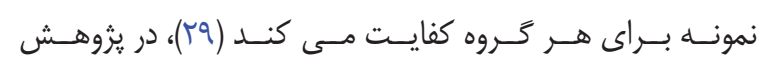

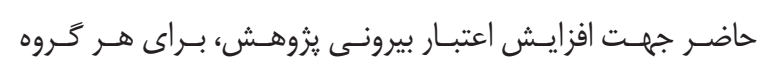

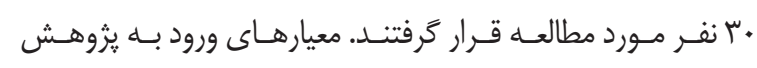

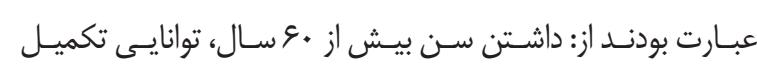

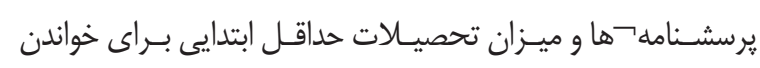

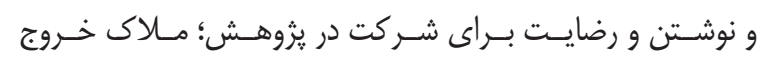

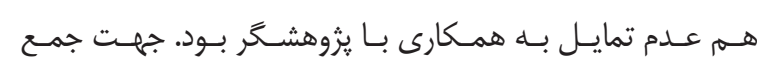

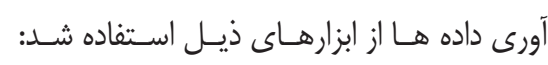

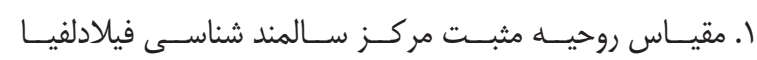
(PGCMS)

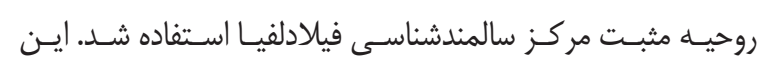

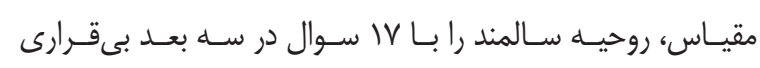

و و رضايـت از سـالمندى، هيجانـات مثبـت و عـدم تنهايسى

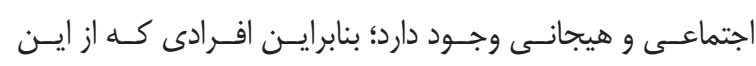

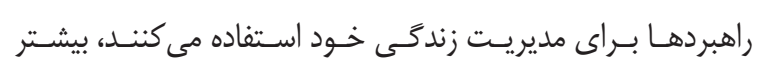

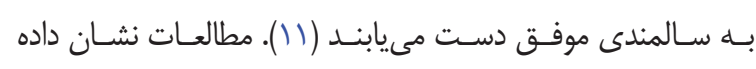

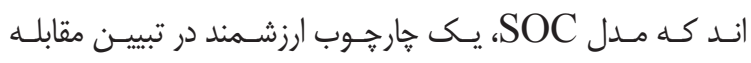

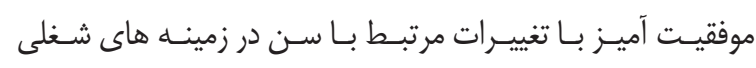

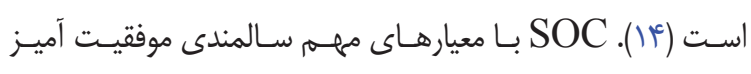

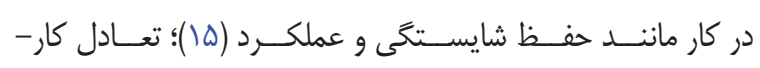

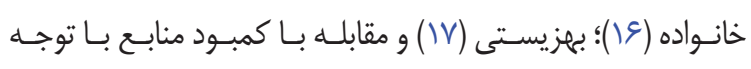

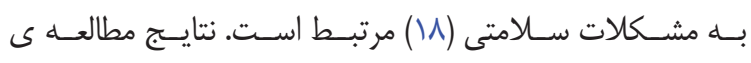

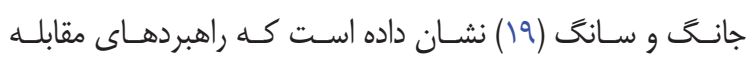

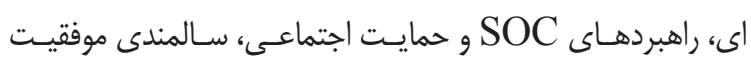

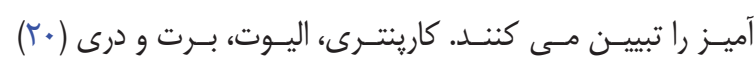

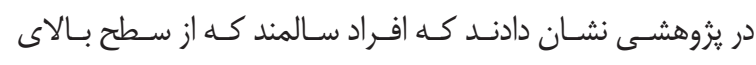

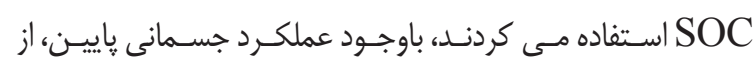

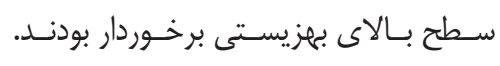

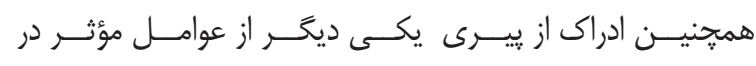

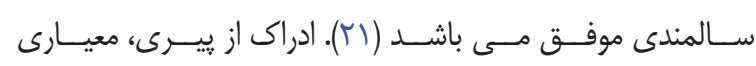

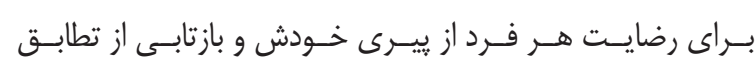

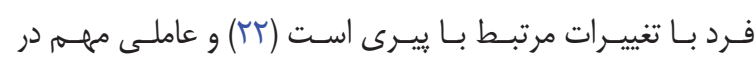

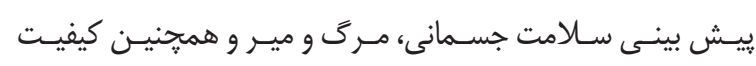

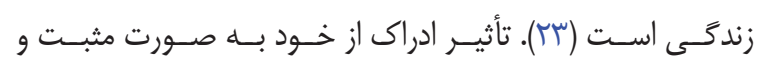
منفـى بـر سـلامت جسـمى و وضعيـت عملكـردى و وحتـى تأثيـر

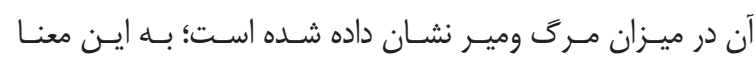

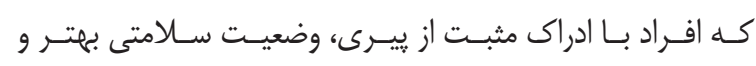

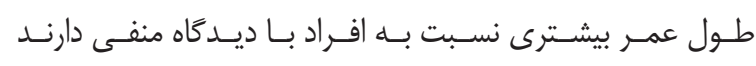

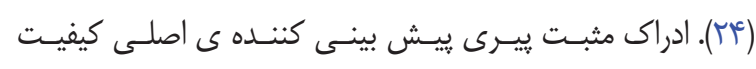

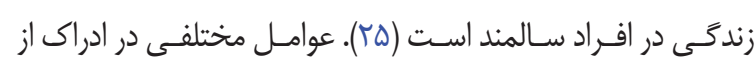

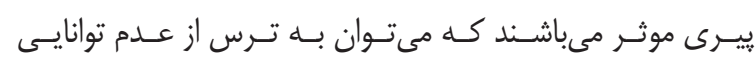

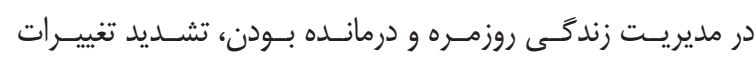

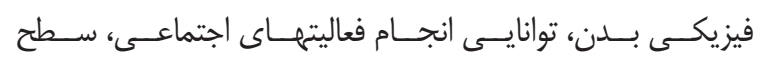

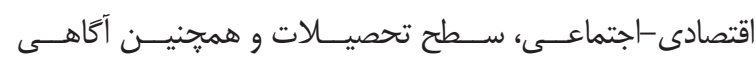

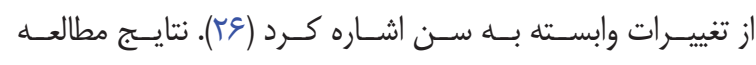

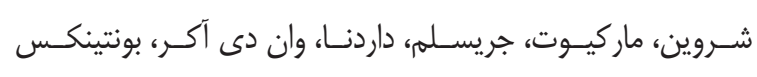

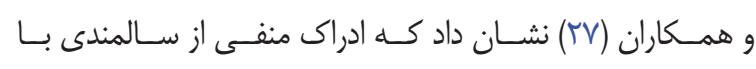

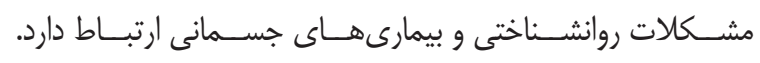

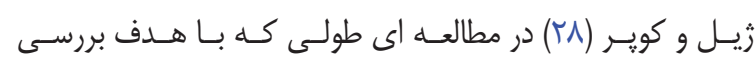

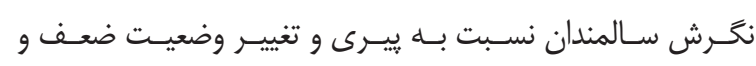




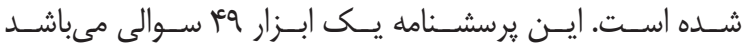

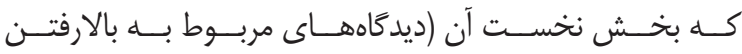

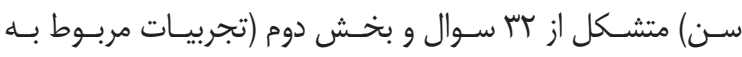

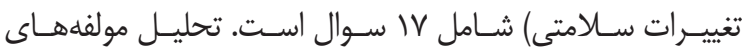

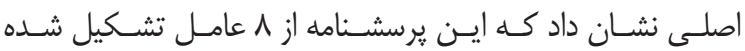

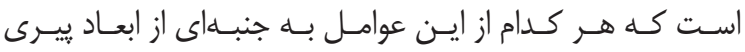

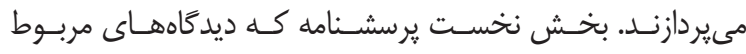

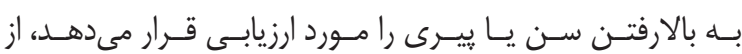

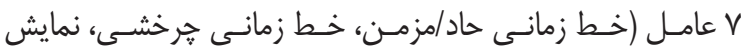

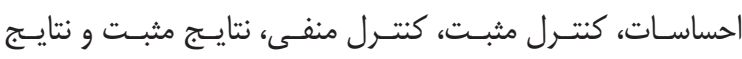

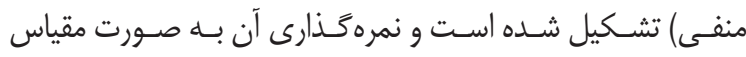

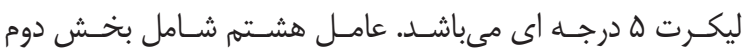

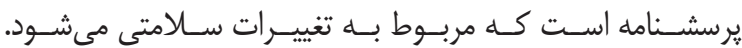

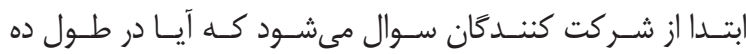

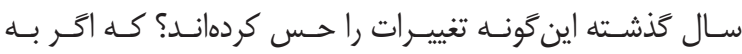

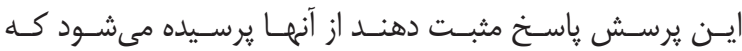

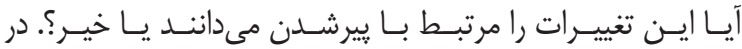

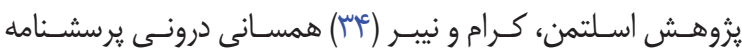

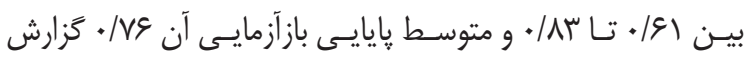

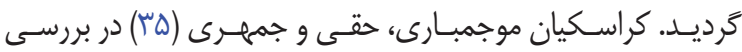

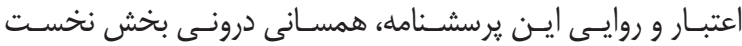

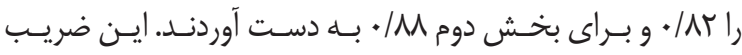

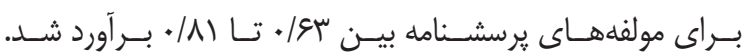

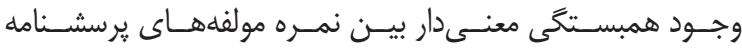

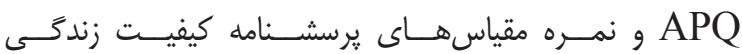

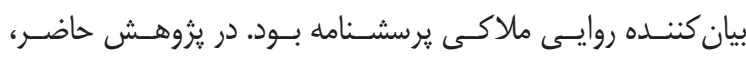

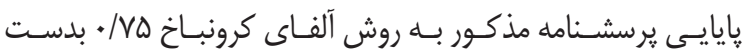

روش خـردآَوى اطلاعـات در ايـن يزوهـش بصـورت ميدانسى بود.

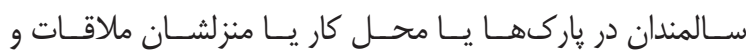

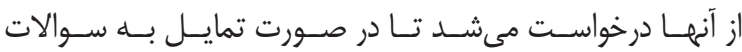

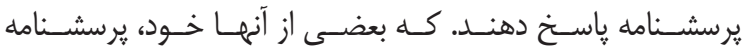

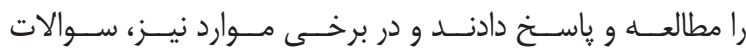

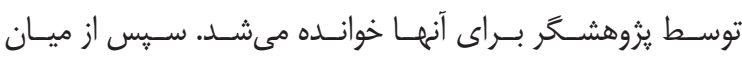

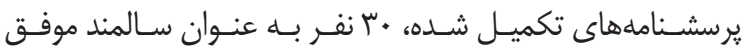

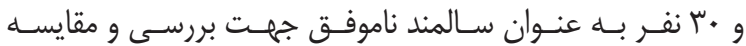

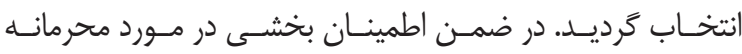

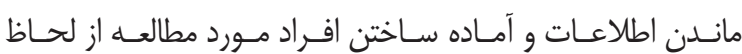

شـامل و سـوال، نارضايتـى نسـبت بــهـ تنهايسى شـامل 9 سـوال

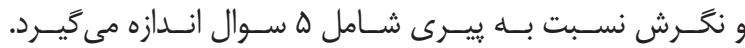

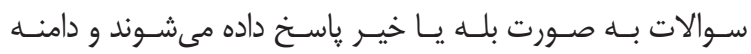

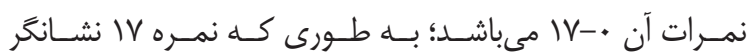

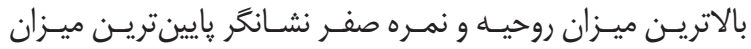

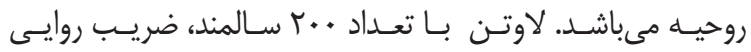

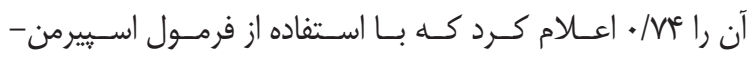

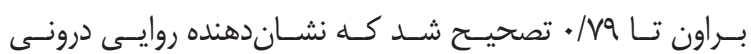

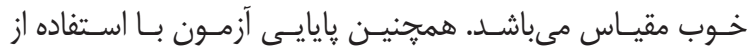

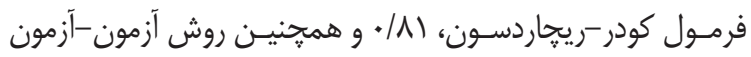

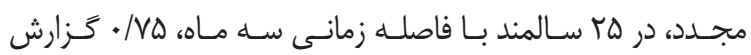

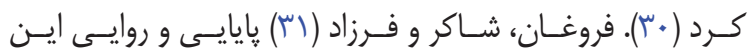

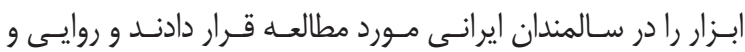

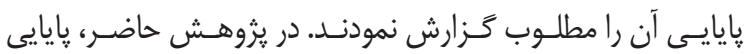

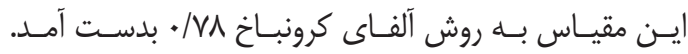

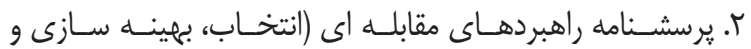

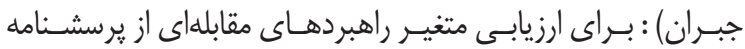

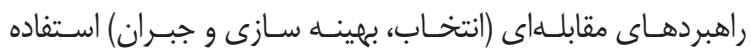

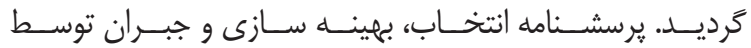

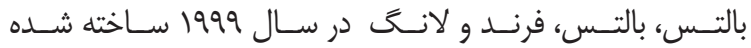

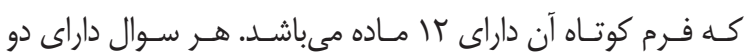

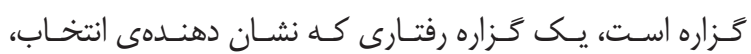

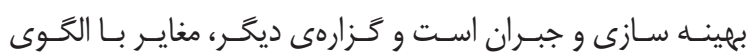

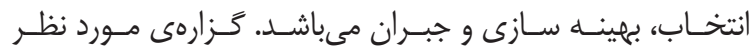

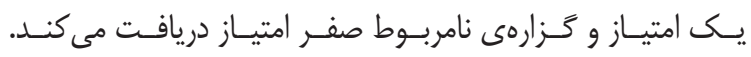

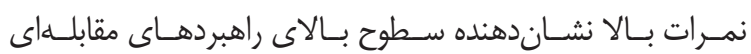

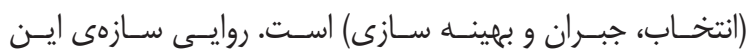

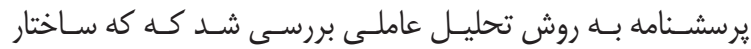

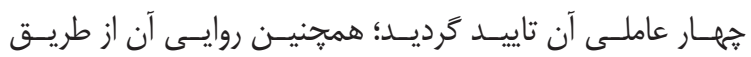

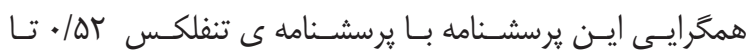

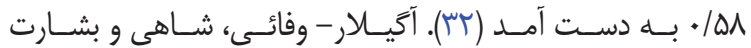

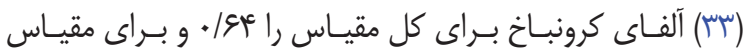

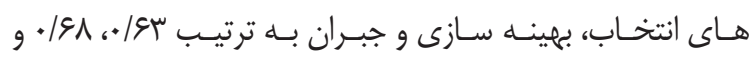

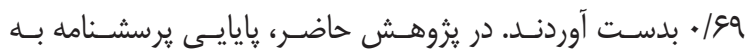

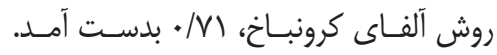

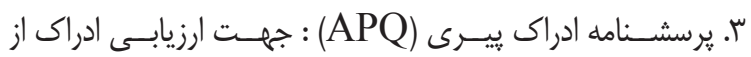

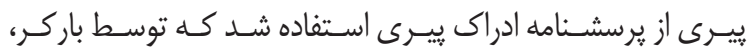

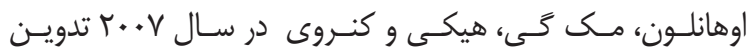


بـراى سـطوح متغيــر مسـتقل برابرنـــ.

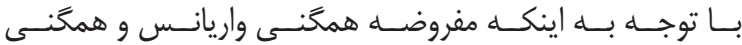

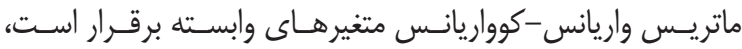

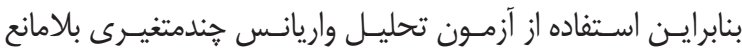

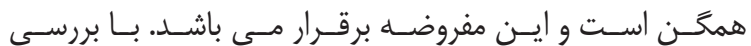

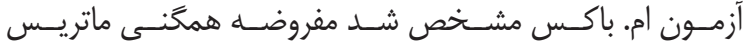

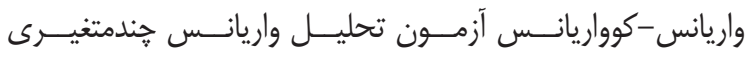

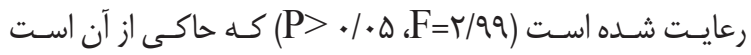

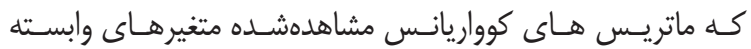

جدول سب: نتايج آزمون معنادارى تحليل واريانس جندمتغيرى براى ادراك از بالارفتن سن و ادراك از سلامتى در سالمندان موفق و ناموفق

\begin{tabular}{|c|c|c|c|c|}
\hline مجذور اتا & $\mathrm{P}$ & $\mathrm{F}$ & مقدار & نام آزمون \\
\hline$. / T / f$ &. $\mid+.1$ & $V / V F^{c}$ & $\cdot|r|$ & اثر ييلايي \\
\hline$. / T / f$ & $.1 . .1$ & $V / V{ }^{c}$ &.$/ v 9$ & لامبدا ويلكز \\
\hline$. / T / f$ & $.1+.1$ & $V / V Y^{e}$ &.$/ T V$ & اثر هتيلنح \\
\hline.$/ T I f$ &. $\mid+1$ & $V / V^{c}$ & $\cdot / T V$ & بزر كترين ريشه خطا \\
\hline
\end{tabular}

بيـن دو حـروه بــا توجـهـ بــهـ متغيرهـاى وابسـته در مجمـوع معنادار

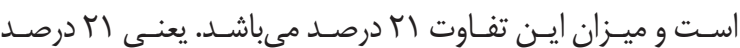

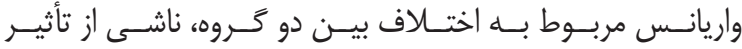

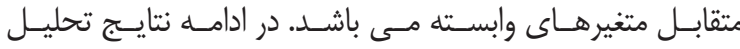

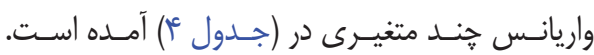

همان طـور كـه در (جـــولَ) مشـاهده مسى شـود آزمـون تحليـل

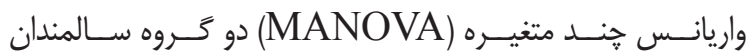

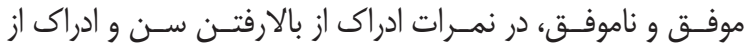

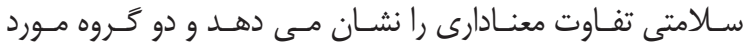

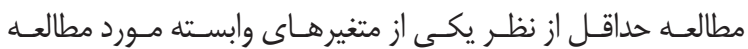

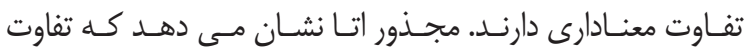
جدول f: نتايج تحليل وارايانس يكراهه بر روى متغيرهاى ادراك از بالارفتن سن و ادراك از سلامتى در سالمندان موفق و ناموفق

\begin{tabular}{|c|c|c|c|c|c|c|}
\hline اندازه تأثير & $\mathrm{P}$ & $\mathrm{F}$ & MS & df & $\mathrm{SS}$ & شاخص \\
\hline $.1 \cdot 1$ & $\cdot / \& \Delta$ & $\cdot \mid \Delta \wedge \varepsilon$ & $\mid \& 9 / 99$ & 1 & $\mid 99 / 99$ & ادراى از بالارفتن سن \\
\hline$\cdot|r|$ & $.1 .+1$ & $10 / 19$ & $195 / 5$. & 1 & $19 \% / \%$. & ادراى از سلامتى \\
\hline
\end{tabular}

در مقايسـهـ بـا سـالمندان راهبردهـاى مقابلـه اي بهتـر و بيشـترى

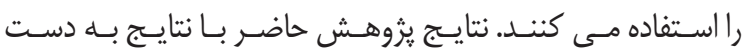

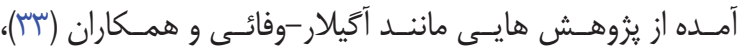

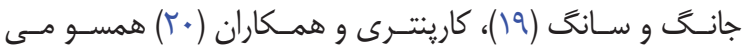

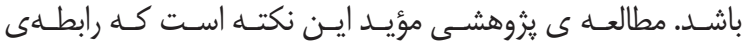

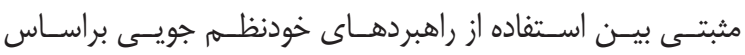

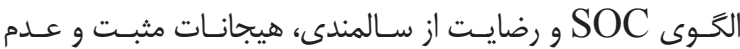
تنهايسى اجتماعى و هيجانسى وجـود دارد؛ بنابرايـن افـرادى كـهـ از ازئ

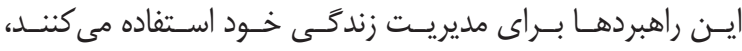

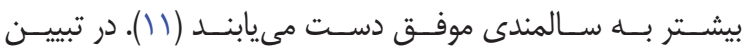

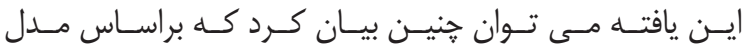

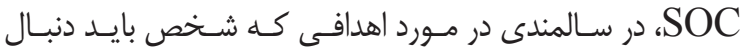

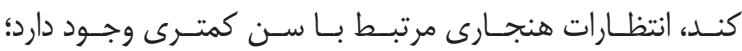

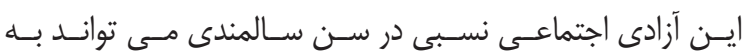

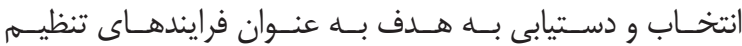
رشــدى، اهميـت بيشـترى ببخشــــ از ســوى ديخــر، سـالمندى

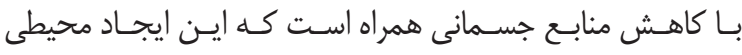

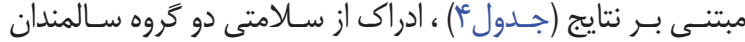

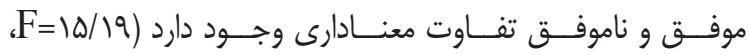

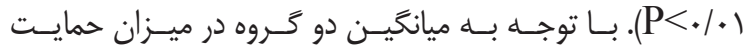

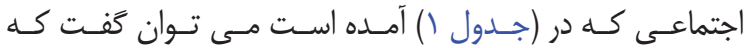

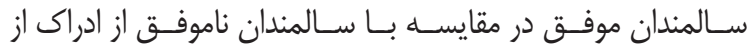

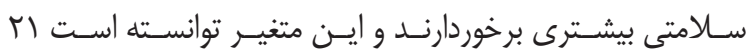

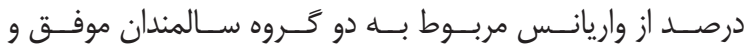

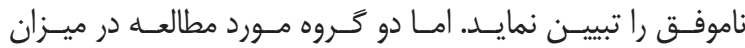

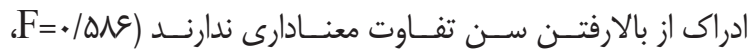
(P>./.0

يزووهـش حاضـر بــاــــــف بررسـى راهبردهــاى مقابلـهاى و ادراك

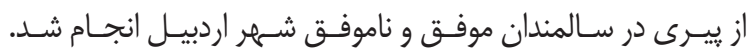

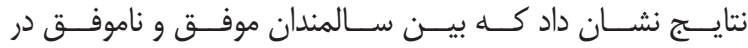

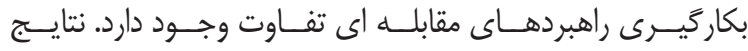

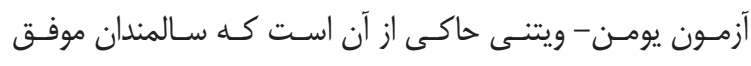




\section{فرشته يور محسنى كلورى هو هكاران}

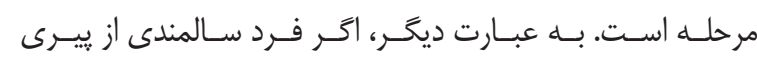

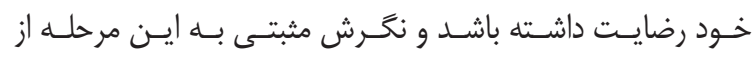

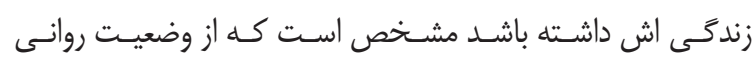

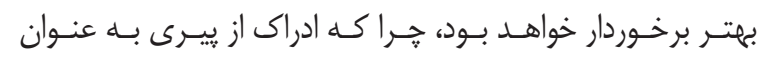

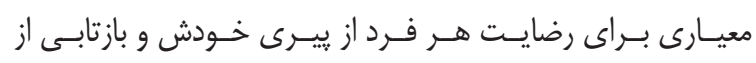

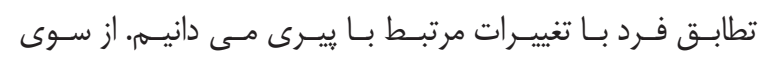

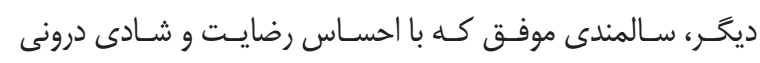

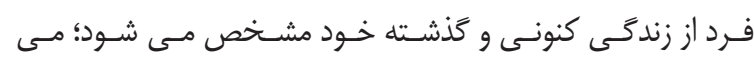

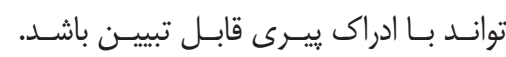

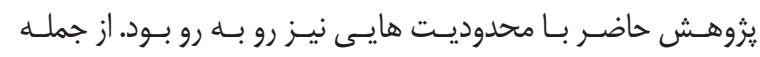

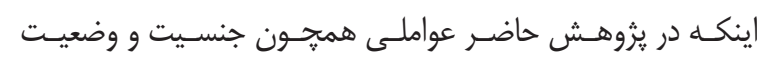

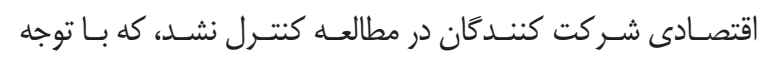

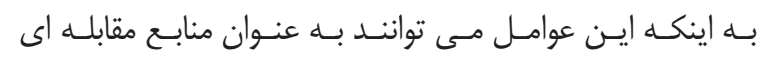

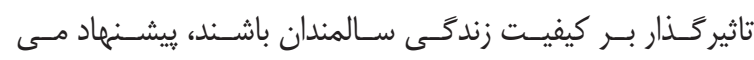

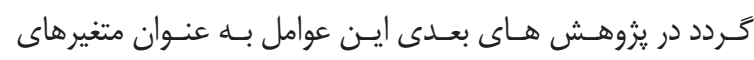

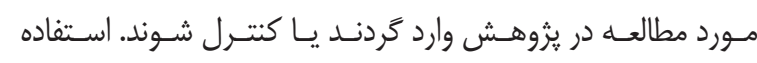

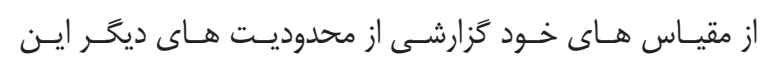

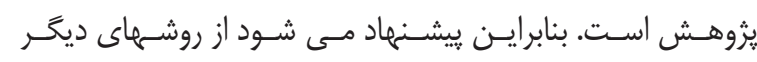

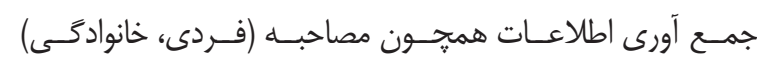

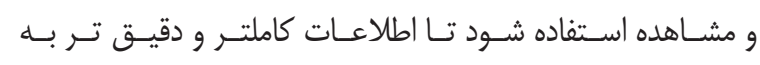

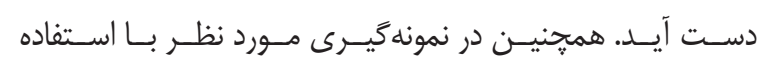

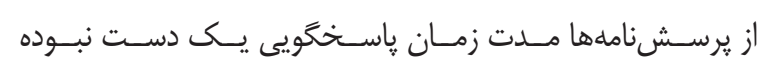

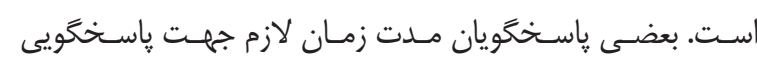

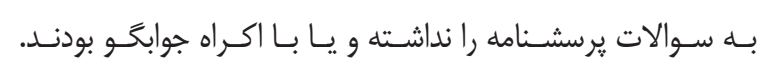

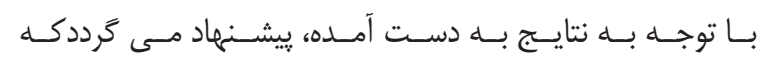

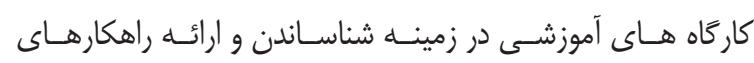

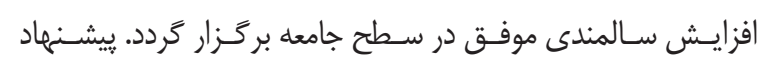

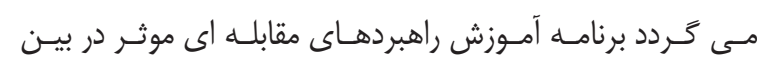

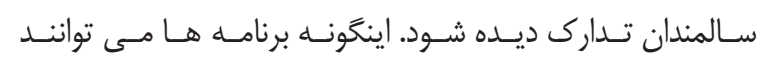

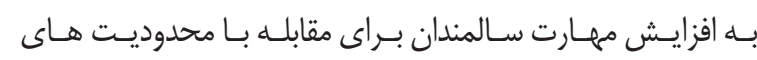

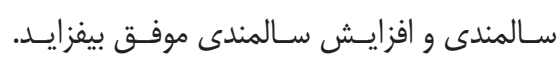

\section{نتيجه كيرى}

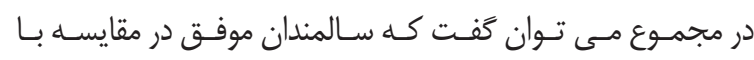

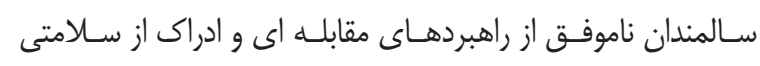

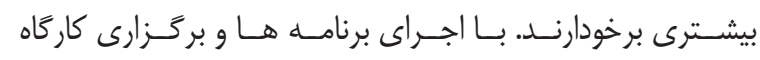

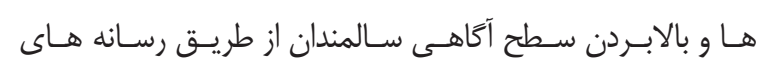

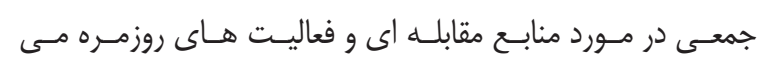

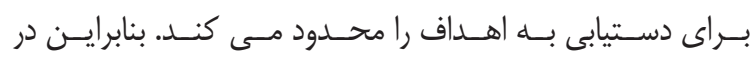

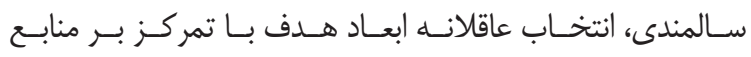

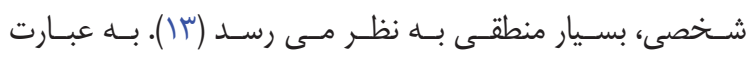

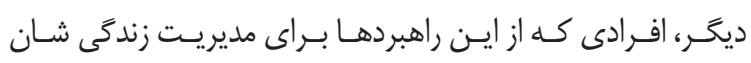

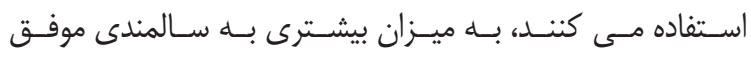

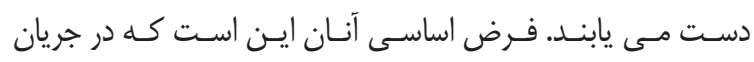

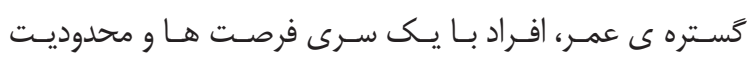

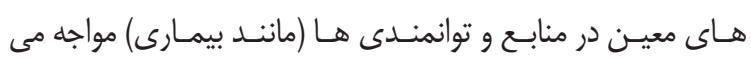

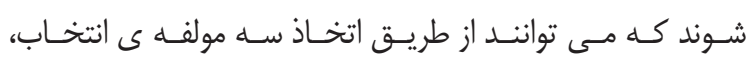

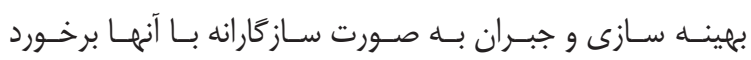

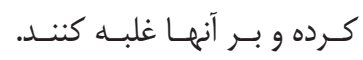

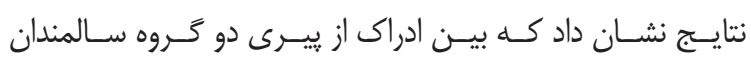

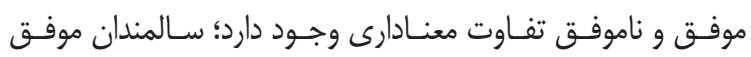

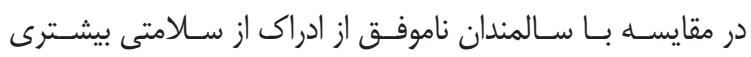

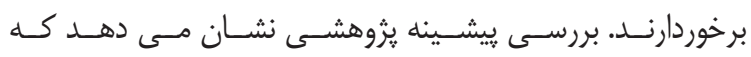

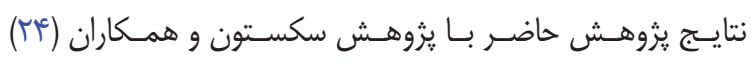

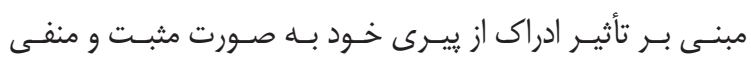

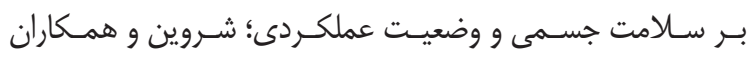

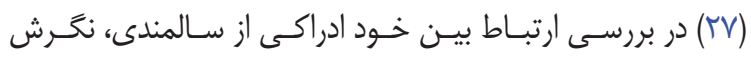

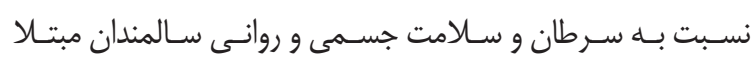

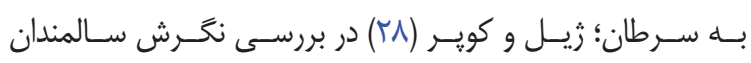

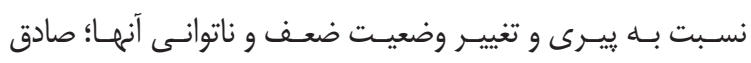

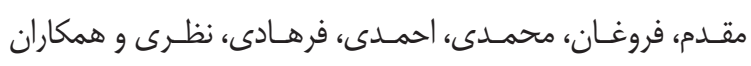

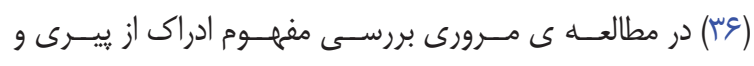

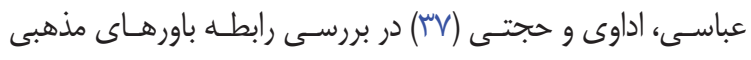

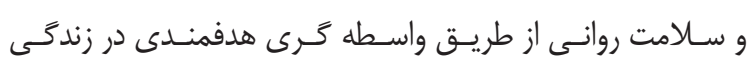

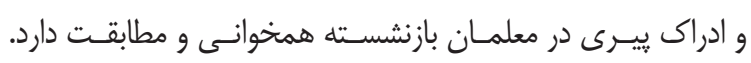

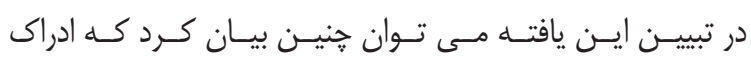

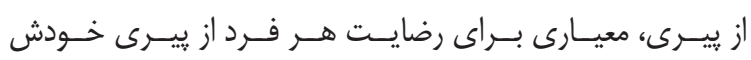

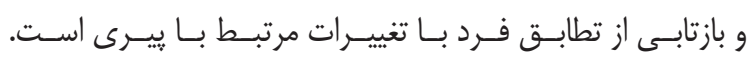

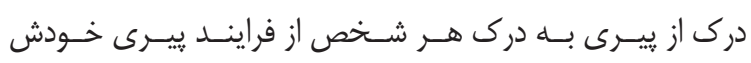

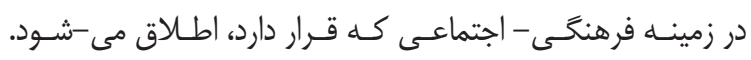

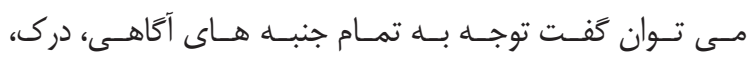

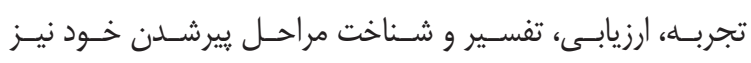

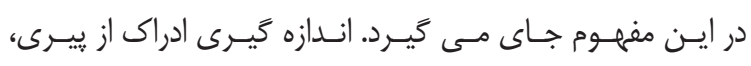

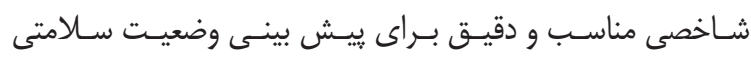

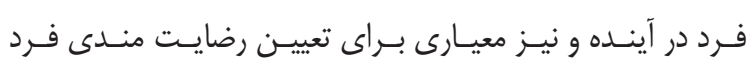

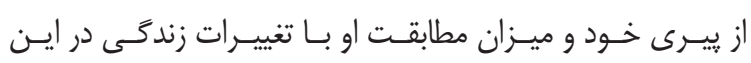




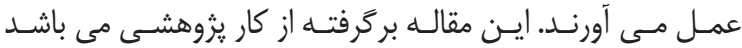

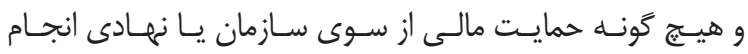

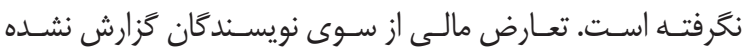

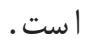

\section{References}

1. Mirzaie M, Darabi S. Population aging in Iran and rising health care costs. Salmand: Iranian Journal of Ageing. 2017; 12 (2):156-69.

2. United Nations, Department of Economic and Social Affairs, Population Division. World Population Prospects: The 2017 Revision, Key Findings and Advance Tables. Working Paper No; 2017. ESA/P/WP/248.

3. Sullivan MP, Crampton A. Population aging and social work practice with older adults: Demographic and policy challenges. International Social Work. 2011; 54 (3):313-29.

4. Fernández-Ballesteros R., Robine JM, Walker A, Kalache A. Active aging: A global goal.Current Gerontology and Geriatrics Research. 2013; 1-4.

5. Bladi Mousavi P, Nasiri N. The situation of the elderly in the country. Statistical Center of Iran; 2018.

6. Rivera-Torres S, Fahey TD, Rivera MA. Adherence to exercise programs in older adults: Informative report. Gerontology \& Geriatric Medicine. 2019; 5: 2333721418823604.

7. Cosco TD, Stephan BCM, Bryne C. On the success of the successful aging paradigm. Journal of Applied Geronto. 2013; 32 (3): 275-6.

8. Berlin K, Kruger T, Klenosky DB. A mixedmethods investigation of successful aging among older women engaged in sports-based versus exercise-based leisure time physical activities. Journal of Woman \& Aging. 2018; 30 (1): 27-37.

9. Panday R, Upadhyay R. Coping responses among elderly living within family setup. Indian Journal of Health Social Work. 2019; 1 (1): 26-30.

10. Nieto M, Romero D, Ros L, Zabala C, Martinez M, Ricarte JJ, et al. Differences in coping strategies between young and older adults: The role of executive functions. The International Journal of Agingand Human Developmen. 2019; 90 (1): 28-49.

11. Kahana E, Kahana B, Lee JE. Proactive approaches to successful aging: One clear path through the forest. Gerontology. 2014; 60:466-
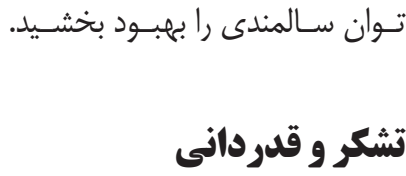

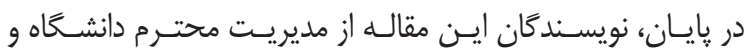

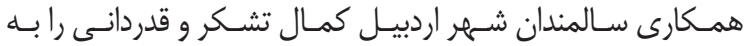
74.

12. SchmittA, Zacher H, Frese M. The buffering effect of selection, optimization, and compensation strategy use on the relationship between problem solving demands and occupational well-being: a daily diary study. Journal of Occupational Health Psychology. 2012; 17 (2): 139-49.

13. Freund AM, Nikitin J, Riediger M. Successful aging. In R. M. Lerner, M. A. Easterbrooks, J. Mistry, \& I. B. Weiner (Eds.), Handbook of psychology: Developmental psychology (p. 615-637). John Wiley \& Sons Inc; 2013.

14. Muller A, Angerer P, Becker A, Gantner M, Gündel H, Heiden B, et al. Bringing successful aging theories to occupational practice: Is selective optimization with compensation trainable. Work, Aging and Retirement. 2018; 4 (2): 161-74.

15. Riedel N, Müller A, Ebener M.Applying strategies of selection, optimization, and compensation to maintain work ability-a psychosocial resource complementing the job demand-control model? Results from the representative lidA cohort study on work, age, and health in Germany. Journal of Occupational and Environmental Medicine. 2015; 57: 552-61.

16. Weber J, Jörres R, Kronseder A, Müller A, Weigl M, Chmelar C. Learning on the job, the use of selection, optimization, and compensation strategies, and their association with telomere length as an indicator of biological aging. International Archives of Occupational and Environmental Health. 2019; 92 (3): 361-70.

17. Zacher H, Frese M. Maintaining a focus on opportunities at work: The interplay between age, job complexity, and the use of selection, optimization, and compensation strategies. Journal of Organizational Behavior. 2011; 32: 291-318.

18. Demerouti E, Bakker AB, Leiter M. Burnout and job performance: The moderating role of selection, optimization, and compensation strategies. Journal of Occupational Health 
Psychology. 2014; 19: 96-107.

19. Jang YM, Song R. Structural equation modeling on successful aging in elders with chronic obstructive poulmonary disease based on selection-optimization-compensation strategy. Journal of Korean Academy of Nursing. 2017; 47(4): 488-98.

20. Carpentieri JD, Elliott J, Brett CE, Deary IJ. Adapting to aging: Older people talk about their use of selection, optimization, and compensation to maximize well-being in the context of physical decline. The Journals of Gerontology. 2017; 72 (2): 351-61.

21. Fathi A, Sarkheil B. View to aging of population in Iran and world. Statistics Journal; 2013;1:2327.

22. Wurm S, Benyamini Y. Optimism buffers the detrimental effect of negative self-perceptions of ageing on physical and mental health. Psychol Health. 2014; 29 (7): 832-48.

23. Wurm S, Tomasik MJ, Tesch-Romer C. On the importance of a positive view on ageing for physical exercise among middle-aged and older adults: cross-sectional and longitudinal findings. Psychology and Health. 2010; 25 (1): 25-42.

24. Sexton E, King- Kallimanis BL, Morgan $\mathrm{K}$, McGee H. Development of the brief ageing perceptions questionnaire (B-APQ): a confirmatory factor analysis approach to item reduction. BMC Geriatrics. 2014; 14: 44.

25. Ingrand I, Paccalin M, Liuu E, Gil R, Ingrand P. Positive perception of aging is a key predictor of quality-of-life in aging people. PLoSONE. 2018; 13 (10):e0204044.

26. Kornadt AE, Bellingtier JA, Levy B. Perceptions of agong: Different concepts, lifespan approaches and predictive relationships. Innovation in Aging. 2018; 2 (1): 747.

27. Schroyen S, Marquet M, Jerusalem G, Dardenne B, Van den Akker M, Buntinx F, et al. The link between self-perceptions of aging, cancer view and physical and mental health of older people with cancer: A cross-sectional study. Journal of Geriatric Oncology. 2017; 8 (1): 64-8.

28. Gale CR, Cooper C. Attitudes to ageing and change in frailty status: The English longitudinal study of ageing. Gerontology. 2018; 64: 58-66.

29. Delaware AS. Research methodology in psychology and educational sciences. Tehran:
Virayesh Pub; 2017.

30. Jennifer AB, Neupert, SD. Negative aging attitudes predict greater reactivity to daily Stressors in older adults. Journals of Gerontology: Psychological Sciences. 2018; 73 (7): 1155-9.

31. Foroughan M, Shakeri F, Farzad V. P478: Psychometrical properties of Philadelphia Geriatric Centre Morale Scale in Iranian elderly. European Geriatrics Medicine. 2014; 5(1):S233.

32. Zhang W, Radhakrishnan K. Evidence on selection, optimization, and compensation strategies to optimize aging with multiple chronic conditions: A literature review. Geriatric Nursing. 2018; 39 (5): 534-43.

33. Aguilar Vafaei ME, Shahi H, Besharat MA. Prediction of Successful Aging based on Life Management Strategies. Journal of Aging Psychology. 2015; 1 (2): 57-71.

34. Slotman A, Cramm JM, Nieboer AP. Validation of the Aging Perceptions Questionnaire Short on a sample of community-dwelling Turkish elderly migrants. Health and Quality of Life Outcomes. 2017; 15: 42.

35. Kraskian-Mujembari A, Haghi-Asgarabadi F, Jomehri F. Determination of the psychometric properties of the Aging Perception Questionnaire. Journal of Research in Behavioural Sciences. 2017; 15 (2): 142-50.

36. Sadegh Moghadam L, Foroughan M, Mohammadi F, Ahmadi F, Farhadi A, Nazari S, et al. Aging perception in older adults. Salmand: Iranian Journal of Ageing. 2016; 10 (4): 202-9.

37. Abbasi M, Adavi H, Hojati M. Relationship between religious beliefs and psychological health through purposefulness intermediation in life and aging perception in retired teachers. Journal of Aging Psychology. 2016; 2 (3):195204. 